\title{
Understanding the Associations between Dietary Antioxidants and Obesity
}

\author{
Dongwoo Ham', Hyojee Joung ${ }^{1,2, *}$ \\ ${ }^{1}$ Institute of Health and Environment and ${ }^{2}$ Department of Public Health, Graduate School of Public Health, \\ Seoul National University, Seoul, Korea
}

Received June 25, 2020

Reviewed July 6, 2020

Accepted July 28, 2020

*Corresponding author

Hyojee Joung

https://orcid.org/0000-0003-1182-7786

Department of Public Health, Graduate School of Public Health, Seoul National University, 1 Gwanak-ro, Gwanak-gu,

Seoul 08826, Korea

Tel: +82-2-880-2716

Fax: +82-2-883-2832

E-mail: hjjoung@snu.ac.kr
Obesity is a major public health concern worldwide. The World Health Organization estimated that more than 650 million adults were obese (body mass index $[\mathrm{BMI}] \geq 30 \mathrm{~kg} / \mathrm{m}^{2}$ ) in $2016^{1}$; according to Korea Health Statistics 2018, published by the Korea Centers for Disease Control and Prevention, the prevalence of obesity $\left(\mathrm{BMI} \geq 25 \mathrm{~kg} / \mathrm{m}^{2}\right)$ among Korean adults was $34.6 \%{ }^{2}$ Over the decades, various intrinsic and extrinsic factors such as genetic characteristics, environmental conditions, and lifestyle behaviors have been reported to have associations with obesity. In particular, diet is considered one of the most significant factors related to obesity status. Numerous studies on associations between obesity and diet have predominantly focused on macronutrient intake because excessive energy intake is a well-known major risk factor of obesity. ${ }^{3}$

However, according to recent biochemical studies, oxidative stress due to reactive oxygen species (ROS) in cells and tissues can induce mutations of DNA or dysfunction of bioactive compounds and thereby increase the risks of obesity and its associated complications such as type 2 diabetes mellitus or metabolic syndrome. ${ }^{4}$ Antioxidants can attenuate oxidative stress by reactions with accumulated ROS. Consequently, they are regarded as having the ability to prevent numerous chronic diseases including obesity. ${ }^{4}$

Antioxidants are categorized into endogenous and exogenous types. ${ }^{4}$ Endogenous antioxidants, such as glutathione peroxidase, superoxide dismutase, and catalase, are produced in the body. ${ }^{4} \mathrm{Ex}$ ogenous antioxidants can be consumed from foods and include flavonoids, carotenoids, tocopherols, ascorbic acid, and selenium. ${ }^{4}$ Flavonoids and carotenoids are plant-based organic components that have been reported as phytochemicals having antioxidant potential. ${ }^{5}$ Flavonoids are subcategorized into flavonols, flavones, flavanones, flavanols, anthocyanidins, isoflavones, and proanthocyanidins. ${ }^{5,6}$ Carotenoids include $\alpha$-carotene, $\beta$-carotene, lycopene, cryptoxanthin, lutein, and zeaxanthin. ${ }^{5,7}$ The major food groups rich in antioxidants are fruits, vegetables, legumes, and whole grains. ${ }^{5,8}$

Several epidemiological studies observed that antioxidant intake was inversely related to obesity. According to our previous research, which analyzed the data of the Korea National Health and Nutrition Examination Survey (KNHANES), women having obesity (BMI $\geq 25 \mathrm{~kg} / \mathrm{m}^{2}$ ) or abdominal obesity (waist circumference $\geq 85 \mathrm{~cm}$ ) consumed significantly less $\alpha$-carotene, retinol, $\alpha$-tocopherol, $\gamma$-tocopherol, and vitamin E (in $\alpha$-tocopherol equivalents) than non-obese women. ${ }^{7}$ Moreover, the highest tertile groups of total carotenoid, $\alpha$-carotene, lycopene, vitamin A (in retinol activity equivalents), $\gamma$-tocopherol, total flavonoid, flavonol, flavanone, fla- 
vanol, isoflavone, and proanthocyanidin intakes had significantly lower odds ratios of abdominal obesity compared with the lowest tertile groups. ${ }^{6,7}$ Zamora-Ros et al. ${ }^{9}$ assessed anthocyanidin intake of participants in the European Prospective Investigation into Cancer and Nutrition study and reported that people with normal BMI level consumed more anthocyanidins and their subclasses (cyanidin, malvidin, pelargonidin, peonidin, and petunidin) than obese people. According to the pooled analyses of three large-scale cohorts in the United States (Health Professionals Follow-up Study, Nurses' Health Study, Nurses' Health Study II), consumption of flavonols, flavanones, flavan-3-ols, anthocyanidins, and flavonoid polymers as well as total flavonoids was significantly related to decreased body weight. ${ }^{10}$ However, Cory et al. ${ }^{11}$ pointed out in their recent review that inverse associations between dietary antioxidants and obesity reported from observational studies should be interpreted with caution because foods rich in antioxidants have relatively few calories per serving size. Therefore, the confounding effects of energy intake should be adjusted in observational studies to verify the associations of dietary antioxidants with obesity. Moreover, further feeding-intervention studies that provide experimental diets containing the same calories but different amounts of antioxidants and compare changes in metabolic indices according to consumption levels of antioxidants are needed.

Meanwhile, McCarty ${ }^{8}$ introduced the phytochemical index (PI) in 2004 as a novel concept for evaluating phytochemical intake. It is calculated as proportion of calories derived from phytochemicalrich foods (e.g., fruits, vegetables, legumes, nuts, and whole grains) divided by total energy intake. A recent cross-sectional study observed that higher PI was related to lower prevalence of abdominal obesity and metabolic syndrome in Koreans. ${ }^{12}$ PI can be an efficient method for assessing consumption of phytochemical-rich foods if there is no available antioxidant database. However, application of PI might be limited in epidemiological studies because it does not reflect intake of non-caloric and phytochemical-rich foods, such as brewed tea, resulting in possible inaccurate measurement of phytochemical consumption. ${ }^{8}$

To date, several intervention studies on the associations between dietary antioxidants and obesity have aimed to investigate the effects of supplementation or fortification of specific antioxidant components on metabolic indices. ${ }^{13-15}$ However, the effects of anti- oxidant consumption from diet may differ from the effects of supplementation or fortification because different antioxidant components can interact with each other and modulate the influences on health outcomes through synergistic or additive effects. ${ }^{5,8,11}$ Moreover, dietary reference intakes (DRIs) for phytochemicals have not been established, and dose-response relationships between phytochemical intake and metabolic indices are not fully understood. Thus, it is necessary to conduct randomized controlled trials that investigate the effects of comprehensive dietary consumption of antioxidants and strengthen scientific findings for establishment of DRIs or dietary guidelines to prevent chronic diseases.

In conclusion, several epidemiological studies have demonstrated an inverse relationship between dietary antioxidants and obesity. As we suggested, further intervention studies may help elucidate the roles of dietary antioxidants in preventing chronic diseases, including obesity, and contribute to improvement of overall health status.

\section{CONFLICTS OF INTEREST}

The authors declare no conflict of interest.

\section{AUTHOR CONTRIBUTIONS}

Study concept and design: all authors; drafting of the manuscript: $\mathrm{DH}$; critical revision of the manuscript: $\mathrm{HJ}$; administrative, technical, and material support: $\mathrm{HJ}$; and study supervision: $\mathrm{HJ}$.

\section{REFERENCES}

1. World Health Organization. Fact sheets: obesity and overweight [Internet]. Geneva: World Health Organization; 2020 [cited 2020 May 20]. Available from: https://www.who.int/newsroom/fact-sheets/detail/obesity-and-overweight

2. Korea Centers for Disease Control and Prevention. Korea Health Statistics 2018: Korea National Health and Nutrition Examination Survey (KNHANES VII-3). Cheongju: Korea Centers for Disease Control and Prevention; 2019.

3. Bray GA, Frühbeck G, Ryan DH, Wilding JP. Management of obesity. Lancet 2016;387:1947-56. 
4. Rupérez AI, Gil A, Aguilera CM. Genetics of oxidative stress in obesity. Int J Mol Sci 2014;15:3118-44.

5. Zhang YJ, Gan RY, Li S, Zhou Y, Li AN, Xu DP, et al. Antioxidant phytochemicals for the prevention and treatment of chronic diseases. Molecules 2015;20:21138-56.

6. Kim SA, Kim J, Jun S, Wie GA, Shin S, Joung H. Association between dietary flavonoid intake and obesity among adults in Korea. Appl Physiol Nutr Metab 2020;45:203-12.

7. Ham D, Kim SA, Jun S, Kang MS, Joung H. Association between antioxidant vitamin intake and obesity among Korean women: using the Korea National Health and Nutrition Examination Survey 2007-2016. J Nutr Health 2018;51:400-13.

8. McCarty MF. Proposal for a dietary "phytochemical index". Med Hypotheses 2004;63:813-7.

9. Zamora-Ros R, Knaze V, Luján-Barroso L, Slimani N, Romieu I, Touillaud M, et al. Estimation of the intake of anthocyanidins and their food sources in the European Prospective Investigation into Cancer and Nutrition (EPIC) study. Br J Nutr 2011; 106:1090-9.

10. Bertoia ML, Rimm EB, Mukamal KJ, Hu FB, Willett WC, Cassidy A. Dietary flavonoid intake and weight maintenance: three prospective cohorts of 124,086 US men and women followed for up to 24 years. BMJ 2016;352:i17.

11. Cory H, Passarelli S, Szeto J, Tamez M, Mattei J. The role of polyphenols in human health and food systems: a mini-review. Front Nutr 2018;5:87.

12. Kim M, Park K. Association between phytochemical index and metabolic syndrome. Nutr Res Pract 2020;14:252-61.

13. Hibi M, Takase H, Iwasaki M, Osaki N, Katsuragi Y. Efficacy of tea catechin-rich beverages to reduce abdominal adiposity and metabolic syndrome risks in obese and overweight subjects: a pooled analysis of 6 human trials. Nutr Res 2018;55: 1-10.

14. Kucukgoncu S, Zhou E, Lucas KB, Tek C. Alpha-lipoic acid (ALA) as a supplementation for weight loss: results from a meta-analysis of randomized controlled trials. Obes Rev 2017; 18:594-601.

15. Marhuenda J, Perez S, Victoria-Montesinos D, Abellán MS, Caturla N, Jones J, et al. A randomized, double-blind, placebo controlled trial to determine the effectiveness a polyphenolic extract (Hibiscus sabdariffa and Lippia citriodora) in the reduction of body fat mass in healthy subjects. Foods 2020;9:55. 